\title{
The structure of Mycobacteria
} 2C-methyl-D-erythritol-2,4-cyclodiphosphate synthase, an essential enzyme, provides a platform for drug discovery

\author{
Lori Buetow ${ }^{1}$, Amanda C Brown ${ }^{2}$, Tanya Parish ${ }^{2}$ and William N Hunter*1
}

Address: ${ }^{1}$ Division of Biological Chemistry and Molecular Microbiology, College of Life Sciences, University of Dundee, Dundee DD1 5EH, UK and ${ }^{2}$ Centre for Infectious Disease, Institute for Cell and Molecular Science, Barts and the London, 4 Newark Street, London E1 2AT, UK

Email: Lori Buetow - l.buetow@dundee.ac.uk; Amanda C Brown - a.c.brown@qmul.ac.uk; Tanya Parish - t.parish@qmul.ac.uk; William N Hunter* - w.n.hunter@dundee.ac.uk

* Corresponding author

Published: 23 October 2007

BMC Structural Biology 2007, 7:68 doi:10.1 186/1472-6807-7-68

This article is available from: http://www.biomedcentral.com/l472-6807/7/68

(C) 2007 Buetow et al; licensee BioMed Central Ltd.

This is an Open Access article distributed under the terms of the Creative Commons Attribution License (http://creativecommons.org/licenses/by/2.0), which permits unrestricted use, distribution, and reproduction in any medium, provided the original work is properly cited.
Received: 8 May 2007

Accepted: 23 October 2007

\begin{abstract}
Background: The prevalence of tuberculosis, the prolonged and expensive treatment that this disease requires and an increase in drug resistance indicate an urgent need for new treatments. The I-deoxy-D-xylulose 5-phosphate pathway of isoprenoid precursor biosynthesis is an attractive chemotherapeutic target because it occurs in many pathogens, including Mycobacterium tuberculosis, and is absent from humans. To underpin future drug development it is important to assess which enzymes in this biosynthetic pathway are essential in the actual pathogens and to characterize them.
\end{abstract}

Results: The fifth enzyme of this pathway, encoded by ispF, is $2 C$-methyl-D-erythritol-2,4cyclodiphosphate synthase (IspF). A two-step recombination strategy was used to construct ispF deletion mutants in $M$. tuberculosis but only wild-type double crossover strains were isolated. The chromosomal copy could be deleted when a second functional copy was provided on an integrating plasmid, demonstrating that isp $F$ is an essential gene under the conditions tested thereby confirming its potential as a drug target. We attempted structure determination of the M. tuberculosis enzyme (MtlspF), but failed to obtain crystals. We instead analyzed the orthologue $M$. smegmatis IspF (MslspF), sharing 73\% amino acid sequence identity, at $2.2 \AA$ resolution. The high level of sequence conservation is particularly pronounced in and around the active site. MslspF is a trimer with a hydrophobic cavity at its center that contains density consistent with diphosphate-containing isoprenoids. The active site, created by two subunits, comprises a rigid CDP-Zn ${ }^{2+}$ binding pocket with a flexible loop to position the $2 C$-methyl-D-erythritol moiety of substrate. Sequence-structure comparisons indicate that the active site and interactions with ligands are highly conserved.

Conclusion: Our study genetically validates $M t l s p F$ as a therapeutic target and provides a model system for structure-based ligand design.

\section{Background}

Approximately one-third of the world's population is infected with Mycobacterium tuberculosis, the causative agent of tuberculosis and 2005, almost 9 million cases of tuberculosis emerged, resulting in an estimated 1.6 million deaths [1]. Typical treatments require combination 
drug therapies taken over a period of 6-9 months. The global economic burden of tuberculosis amounts to approximately $\$ 12$ billion annually. The need for novel chemotherapeutics in the treatment of infection by $M$. tuberculosis is clearly demonstrated by its high infectivity rate and prolonged and extensive therapy requirements.

The isoprenoid biosynthesis pathways are attractive, established targets for chemotherapeutic treatment $[2,3]$. Isoprenoids are building blocks for several biologically or commercially important compounds, including steroids, flavoring compounds like limonene, and natural medicinal products like taxol [4]. Cells are dependent on isoprenoid derivatives for critical functions like growth, hormone-based signaling, differentiation, maintenance of homeostasis, and electron transport in respiration and photosynthesis [4]. In Mycobacteria species, isoprenoid biosynthesis is particularly important for the synthesis of the cell wall, including mycolic acids and lipoarabinomannan [5]. The universal precursors of isoprenoids are the isomers isopentenyl pyrophosphate (IPP) and dimethylallyl pyrophosphate (DMAPP). Synthesis of these precursors occurs via two distinct biochemical pathways. In mammals, fungi, the cytoplasm of plants, and archaebacteria, synthesis occurs via the mevalonate pathway [6], and, in chloroplasts, algae, cyanobacteria, apicomplexa and most eubacteria (including M. tuberculosis), via the 1-deoxy-D-xylulose 5-phosphate (DOXP) or nonmevalonate pathway [7-11]. Fosmidomycin is an inhibitor of the third enzyme in the DOXP pathway, 1-deoxy-Dxylulose 5-phosphate reductoisomerase, and has been used against infections by Plasmodium species $[3,12]$. Since the compound is a clinically approved antibacterial agent then there is chemical validation of this stage of the pathway for drug development. Recently, the crystal structure of the $M$. tuberculosis reductoisomerase has been determined opening up routes to structure-based inhibitor discovery methods targeting that particular stage of the pathway [13].

Eight enzymes are involved in the synthesis of IPP and DMAPP via the DOXP pathway $[8,14]$. IspF, or $2 C$ methyl-D-erythritol-2,4-cylodiphosphate (MECDP) synthase, is the fifth enzyme of the pathway. Structural and biochemical studies, in particular on the Escherichia coli enzyme (EcIspF), demonstrate that IspF directs an intramolecular attack of the 2-phosphate on the internal $\beta$-phosphate of the substrate, 4-diphosphocytidyl-2Cmethyl-D-erythritol-2-phosphate (CDP-ME2P), to form MECDP and CMP (Figure 1). IspF depends on two divalent cations to orient and polarize the substrate during catalysis [15-18] In Gram-negative bacteria and Mycobacteria species, is $p F$ is found in a putative operon with is $p D$, which encodes the third enzyme in the DOXP pathway [14]. Genetic studies indicate that ispF is essential in E. coli as well as Bacillus subtilis $[8,19,20]$ and partial depletion of $i s p F$ in these bacteria increases sensitivity to cell wallactive antibiotics [19]. In larger genomic scale hybridization studies, failure to insert a transposon into the ispF gene also suggests it is essential in Haemophilus influenzae [21] and M. tuberculosis [22].

These observations, in conjunction with the absence of this enzyme from humans, demonstrate the importance of IspF as a novel target for drug discovery. The structure of EcIspF has provided a model for rational ligand design [23] and a high throughput screen has been developed to enable ligand discovery [24]. Little information is available for M. tuberculosis IspF (MtIspF) and earlier genetic studies only suggest that ispF is essential in this organism. Here, we prove that ispF is essential in M. tuberculosis. Furthermore, for use as a model in structure-based ligand

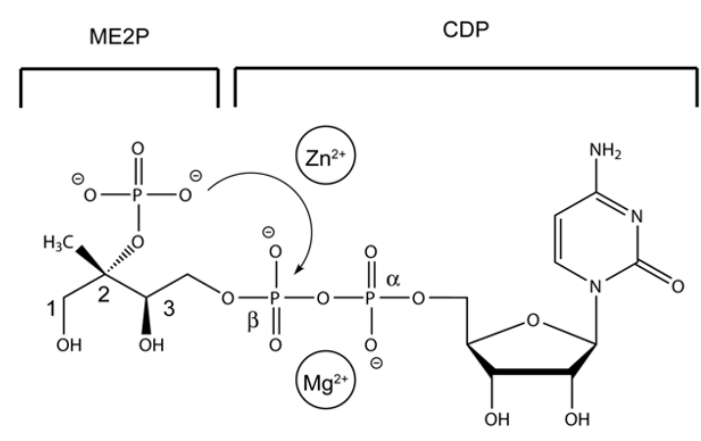

4-diphosphocytidyl-2C-methyl-D-erythritol-2-phosphate (CDP-ME2P)
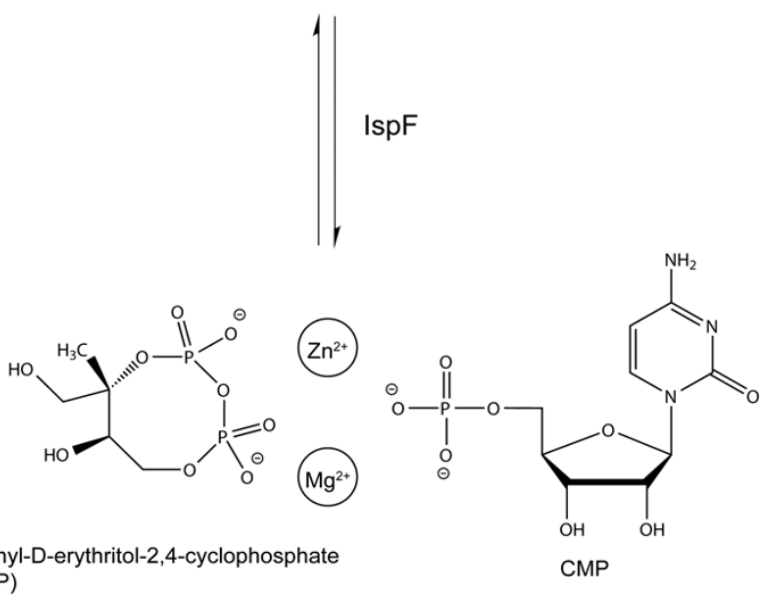

(MECDP)

\section{Figure I}

The IspF reaction. IspF catalyzes the formation of $2 C$-methylD-erythritol 2,4-cyclodiphosphate (MECDP) and CMP by an internal direct attack of the 2-phosphate group on the $\beta$ phosphate of the substrate, 4-diphosphocytidyl-2C-methyl-Derythritol-2-phosphate (CDP-ME2P). The reaction is dependent on divalent cations $\left(\mathrm{Zn}^{2+}\right.$ and under physiological conditions $\mathrm{Mg}^{2+}$ ). 
design, we present a structure of the orthologue, Mycobacterium smegmatis IspF (MsIspF), bound to CDP.

\section{Results and discussion IspF essentiality in $\mathrm{M}$. tuberculosis}

We exploited our previously described methods [25-29] to determine whether ispF was essential in M. tuberculosis. Initially, we attempted to construct a knockout mutant using a two-step homologous recombination procedure. The two-step method employed the use of a suicide (nonreplicating) construct containing an in-frame deletion of the ispF gene (Figures 2, 3). The construct (p2NIL- $\Delta$ ispF) was introduced into wild-type $M$. tuberculosis and single crossover (SCO) recombinant strains obtained. One SCO strain was used to isolate double crossover (DCO) recombinants; in the absence of antibiotic selection, DCO recombinants could have either the wild type or the deletion alleles. We screened 24 DCO recombinants; all had the wild-type gene.

The failure to isolate a deletion strain suggested that ispF is essential in axenic culture. To verify this hypothesis, we made a merodiploid strain in which an additional functional copy of is $p D$ and $i s p F$ were introduced into the SCO strain on an L5-derived integrating vector under the control of the mycobacterial antigen 85A promoter (pAPA3ispDF). The resulting strain had one deleted and two functional copies of ispF. Double crossovers generated from the merodiploid strain were isolated as before. Screening by PCR demonstrated that 19/24 DCOs had the wild-type gene and 5/24 had the deletion allele ( $p=0.04$, Fisher's exact t-test). The genotypes of the transformants with the deletion allele were confirmed by Southern hybridization (Figure 3). Since the chromosomal copy of ispF could only be deleted when a second functional copy was provided, this proved the essentiality of ispF in M. tuberculosis.

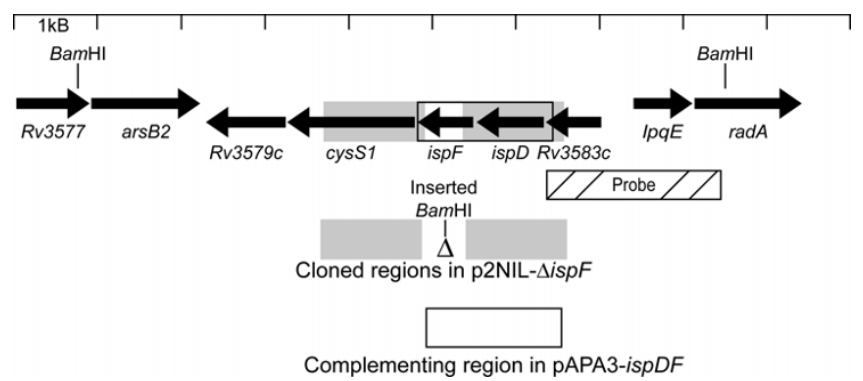

\section{Figure 2}

M. tuberculosis ispF is essential. Map of the ispF genomic region in the wild type and the deletion allele. Regions amplified for the delivery and complementing vectors, restriction sites (intragenic and introduced) and probe location are indicated.

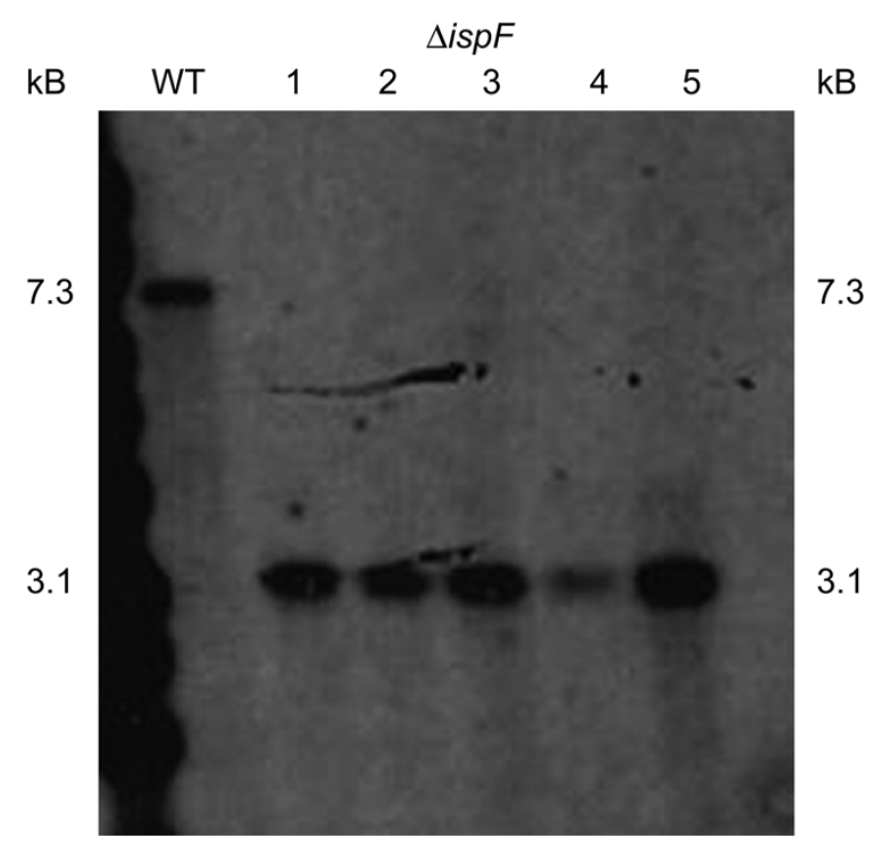

Figure 3

Southern blot of DispF complemented with pAPA3-ispDF (lanes 2-5) and WT (lane I)- DNA was digested with BamHI and probed with an upstream region of ispF. Expected size of WT band was $7.3 \mathrm{~kb}$; DispF mutants were shown to only to possess the deletion band ( $3.1 \mathrm{~kb})$, thus confirming the deletion of ispF.

\section{A Mycobacteria model for structure-based studies}

That ispF is essential in $M$. tuberculosis validates the encoded enzyme as a chemotherapeutic target. We tried to determine the structure of $M t \mathrm{IspF}$ to aid in rational ligand design, but the protein, though efficiently produced in recombinant form, was recalcitrant to crystallization. MtIspF has $73 \%$ amino acid identity to MsIspF, so we chose to study the orthologue on the basis that it would provide a suitable model of the pathogen enzyme. The recombinant $M s I s p F$ is produced in high yield (approximately $30 \mathrm{mg} \mathrm{L}^{-1}$ of bacterial culture), can be purified readily and provided well-ordered single crystals. A surface model of $M s I s p F$, which is colored by shared identity with MtIspF, highlights the strong resemblance between these sequences, particularly at the active site (Figures 4, 5, $6)$. Generally, an accurate homology model is attainable in high sequence identity $(>60 \%)$ cases $[30,31]$ and the use of such models has been successful in structure-based ligand design. In certain cases, even with $<60 \%$ sequence identity, homology models have been found useful. Examples being human carbonic anhydrase [32] and Rho kinase [33] where models were constructed from sequences that shared only $38 \%$ and $37 \%$ identity, respectively. 


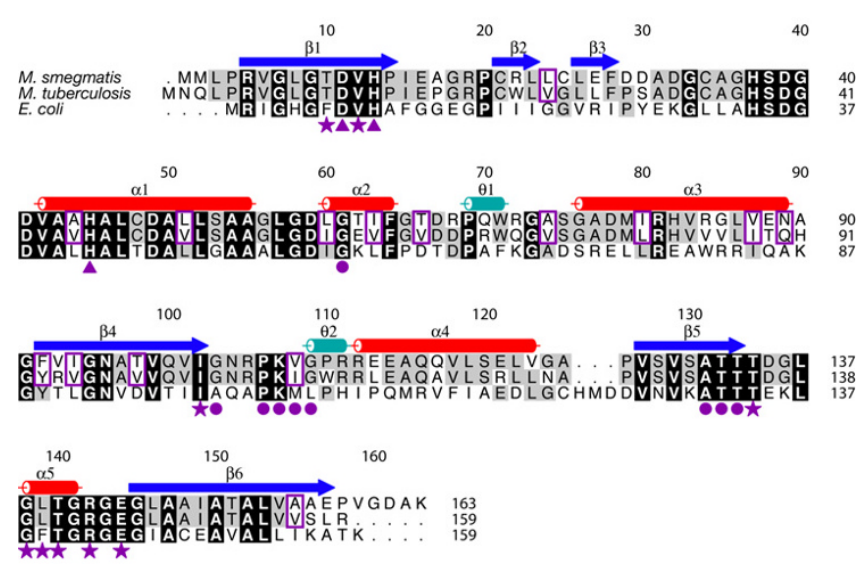

Figure 4

IspF homology. Amino acid sequence alignment of MslspF, MtlspF, and EclspF. Secondary structure elements of MslspF are shown above the sequence. $\beta$-strands are blue, $\alpha$-helices red, and $3_{10}$-helical segments aquamarine. MslspF and EclspF are aligned based on a structural overlay. Residues boxed in black are strictly conserved and those in grey are identical in two of the three sequences; similar residues from the Mycobacteria sequences are outlined in a purple box. $\mathbf{\Delta}$ identifies residues that interact with $\mathrm{Zn}^{2+}, \tau$ with $\mathrm{Mg}^{2+}, \mathrm{H}$ with CDP, and $\checkmark$ with the $2 C$-methyl-D-erythritol moiety of substrate; $\lambda$ denotes residues that line the hydrophobic cavity. The residues that bind $\mathrm{Mg}^{2+}$ and the ME2P fragment of substrate are based on observations in EclspF.

\section{Overall structure}

The structure of $M s$ IspF bound to CDP was determined to a resolution of $2.2 \AA$. There are three subunits (chains A, $\mathrm{B}$, and $\mathrm{C}$ ) in the asymmetric unit, forming a homotrimer about a non-crystallographic axis. The model comprises residues 3-157 for each subunit, with residues 36-37 absent in chains A and B. Structures of several ligandbound and native forms of IspF from Campylobacter jejuni, E. coli,H. influenzae, Shewanella oneidensis, and Thermus thermophilus are available in the Protein Data Bank [PDB, [15,34-37]]. EcIspF [PDB code 1GX1, [16]] was chosen as the model for the structural comparisons to follow because it was built using high-resolution data (1.8 $)$ and contains the ligand CDP. The two sequences share $38 \%$ identity, and the r.m.s.d. values for the superposition of the MsIspF onto the EcIspF trimer range from 1.10-1.16 $\AA$, depending upon which chains are aligned.

MsIspF closely resembles EcIspF [16-18]. Each subunit displays an $\alpha / \beta$ fold which contains six $\beta$-strands, five $\alpha$ helices, and two $3_{10}$ helices (Figures $4,7,8$ ). Four of the strands $(\beta 1, \beta 4-6)$ comprise a central $\beta$-sheet that packs against the $\alpha$ - and $3_{10}$ helices. The other two strands form a short sheet at the end of a loop that extends into the space between $\alpha 1, \alpha 3$, and $\alpha 4$. One $3_{10}$ helix $(\theta 2)$ of

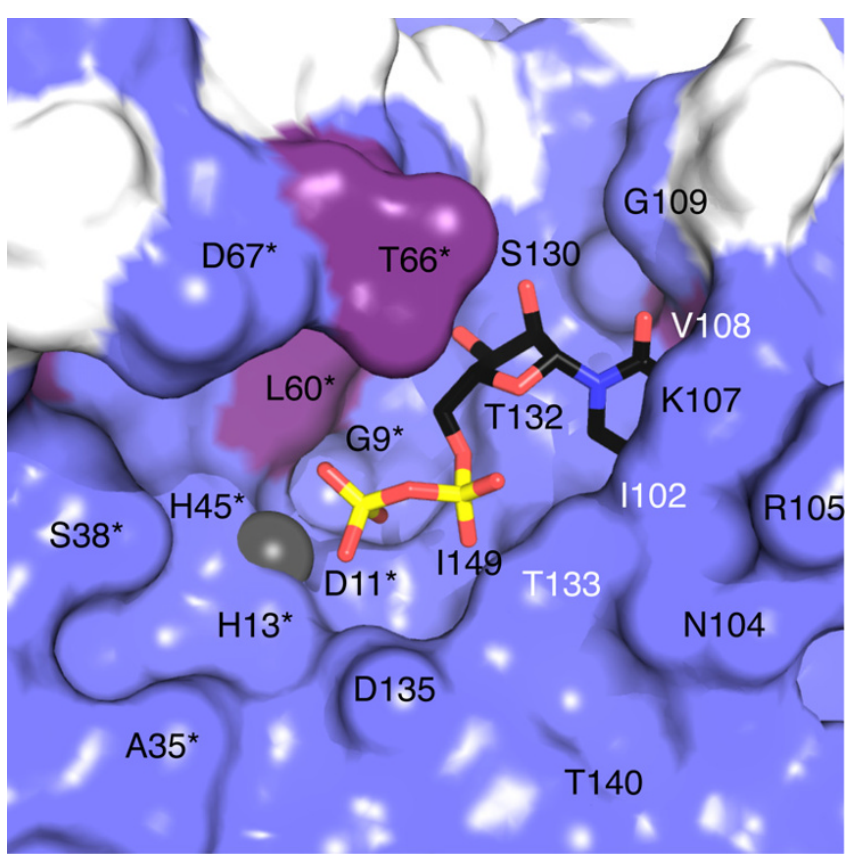

\section{Figure 5}

The van der Waals surface of the active site colored according to shared sequence identity with MtlspF. Identical residues are colored slate-blue and similar residues are purple. The active site $\mathrm{Zn}^{2+}$ is a grey sphere, and CDP is shown as a stick model with $\mathrm{C}$ atoms in black, $\mathrm{N}$ blue, $\mathrm{O}$ red, and $\mathrm{P}$ yellow. An asterisk indicates contributions from an adjacent subunit.

MsIspF overlays with that of EcIspF (designated $\theta 1$ by [16]), but the second ( $\theta 1)$ occurs between $\alpha 2$ and $\alpha 3$ rather than following $\alpha 4$.

Trimer formation arises from edge-to-face packing of the $\beta$-sheets, with the largest section of the interface occurring between $\beta 1$ and $\beta 5$ of adjacent subunits (Figures 7,8 ). Thus, the interior shape of the trimer resembles a trigonal prism whose faces are comprised of $\beta$-sheets from the individual subunits. The MsIspF trimer has the same overall dimensions as EcIspF, measuring approximately $40 \AA$ in height along the three-fold symmetry axis and $60 \AA$ in diameter at the widest point perpendicular to this axis. In addition, like the E. coli enzyme, most of the hydrogen bonds between the subunits involve side chain interactions. The trimer interface interactions also resemble those of $E$. coli in that they are primarily hydrophobic; approximately $65 \%$ of atoms comprising both of these enzyme interfaces are non-polar. E. coli and M. smegmatis are mesophiles. In contrast, only $58 \%$ of atoms in the interface of IspF from the thermophile T. thermophilus are non-polar [35]. 

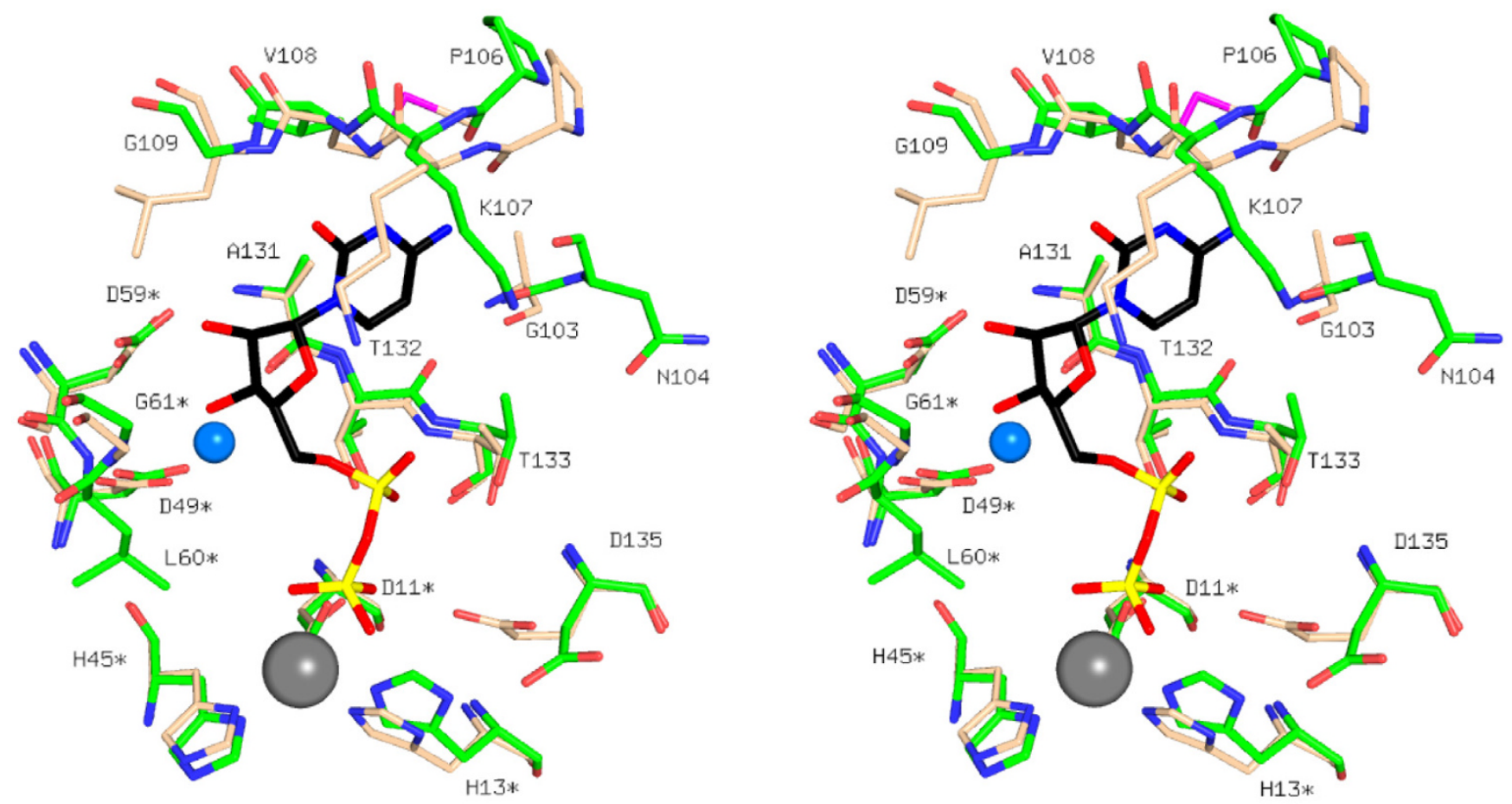

Figure 6

Stereo-view of the active site overlay of MslspF and EclspF. Residues from MslspF are labeled and CDP-B is the depicted conformer. Protein atoms are colored: $\mathrm{C}$ of MslspF green, $\mathrm{C}$ of EclspF wheat, all $\mathrm{N}$ atoms blue, $\mathrm{O}$ atoms red, and Se atoms magenta. The marine sphere depicts the water molecule that contributes to solvent-mediated interactions between IspF and the ribose hydroxyls of CDP.

\section{Hydrophobic cavity}

At the center of the trimer is a hydrophobic cavity that opens toward the C-terminal ends of $\beta 1, \beta 4$ and $\beta 5$. Side chains of residues Thr10, Val12, Ile102, Thr134, Leu139 and Thr140 from each subunit line the interior of the cavity while two arginines (Arg142 from subunits A and B) and the main chain of Gly138 and Leu139 of subunit $C$ shape the aperture (data not shown). Arg142 is held in place through an electrostatic attraction to Glu144. In EcIspF, a salt-bridge between Arg142-Glu144 from all three subunits forms the aperture. Here, subunit $\mathrm{C}$ is less ordered and this contributes to the observed asymmetry. The density is poorly defined between residues 137-144 in subunit $\mathrm{C}$ and the average thermal parameter for this region $\left(58.4 \AA^{2}\right)$ is much higher than in subunits A (34.6 $\AA^{2}$ ) and $\mathrm{B}\left(18.1 \AA^{2}\right)$.

The distance from the base of the cavity to the opening (16 $\AA$ ) and the diameter of the aperture ( $6 \AA)$ are comparable to those observed in $E c I s p F$. The volume of the cavity of $M s \operatorname{spF}\left(1940 \AA^{3}\right)$, however, is significantly larger than that of EcIspF (1540 $\AA^{3}$ ). In EcIspF the cavity is ellipsoidal and the floor parabolic; the major axis of the ellipsoid runs from the aperture to the floor of the cavity [15]. In $M s I s p F$ the cavity is trigonal pyramidal, with the aperture corresponding to the tip of the pyramid and the floor to the base. Residue differences in the lining of the cavity contribute to shape and diameter variation. In E. coli, the cavity is lined with the side chains of six large hydrophobic residues, Phe7 and Phe139 from each subunit, whereas the corresponding residues in MsIspF are Thr10 and Leu139. In EcIspF, the floor of the cavity is sealed by three His5-Glu149 salt bridges [15]. Hydrophobic interactions seal the floor of MsIspF. Here, residues Leu8 and Ile149 replace the EcIspF salt-bridge. The cavity in MtIpsF should bear a strong resemblance to that in MsIspF since the residues that contribute to the lining (discussed above) are strictly identical in the two sequences (Figure 4).

In common with crystal structures of other IspF trimers, non-protein electron density was observed in the hydrophobic cavity of MsIspF. In EcIspF, phosphate, farnesyl pyrophosphate, GPP, and IPP have been shown to bind within this cavity [15]. There is as yet no evidence to prove that ligand binding here regulates enzyme activity. The 


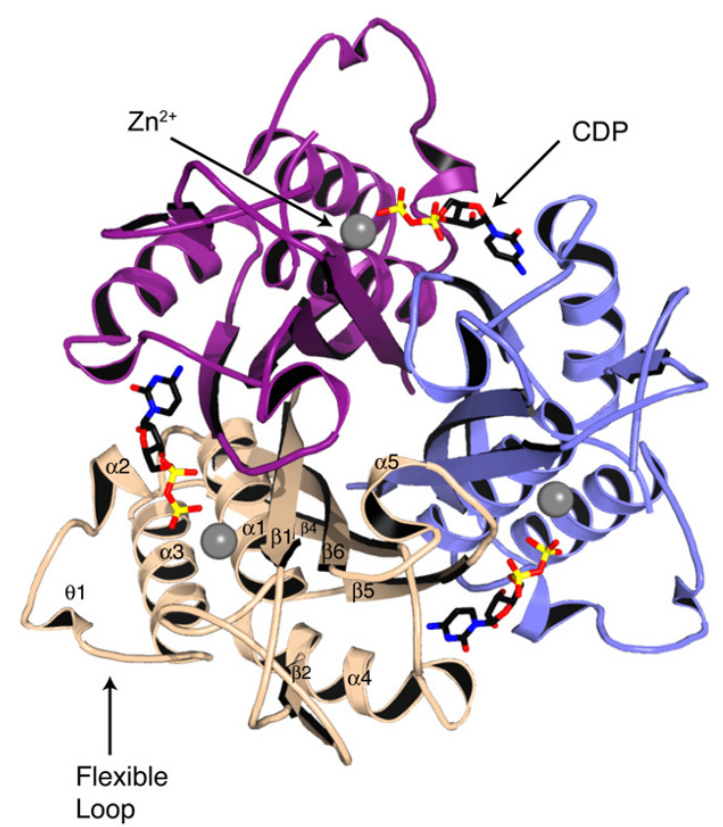

Figure 7

Ribbon diagram of the trimer. The MslspF trimer viewed down the molecular three-fold axis. The individual subunits are shown in slate, wheat, and purple. Selected secondary structure elements of the wheat subunit, CDP and $\mathrm{Zn}^{2+}$ are depicted as in Figure 5.

cavity is distant from the three catalytic sites but since, as will be explained, oligomerisation is required to generate the functional enzyme then occupancy of the hydrophobic cleft may contribute to the stability of the IspF trimer.

In MsIspF the density observed in the cavity is diffuse and we presume that a similar mixture of ligands may be present. IPP was modeled into this density at 50\% occupancy based on fit and ligand identification in the EcIspF cavity. Although a methodical and thorough approach was used in fitting the ligand, the thermal parameters of IPP $\left(47.5 \AA^{2}\right)$ exceed the average of the protein $\left(27.5 \AA^{2}\right)$. The ligand-protein interactions, though not clearly defined, do resemble those observed in EcIspF [PDB code $1 \mathrm{H} 47,[15]]$. The guanidino groups of Arg142 from two subunits bind to the $\beta$-phosphate; in $E c I s p F$, the side chain from the corresponding residue (Arg142) of all three subunits contributes to this interaction. In $M s I s p F$, the bridging phosphodiester oxygen of IPP binds to the amide of Leu139 in subunit $\mathrm{C}$ and one of the $\alpha$-phosphate oxygens binds to the main chain amide of Leu139 in subunit B. In EcIspF, these ligand atoms interact with the main chain amide of the corresponding residue (Phe139) of all three subunits.

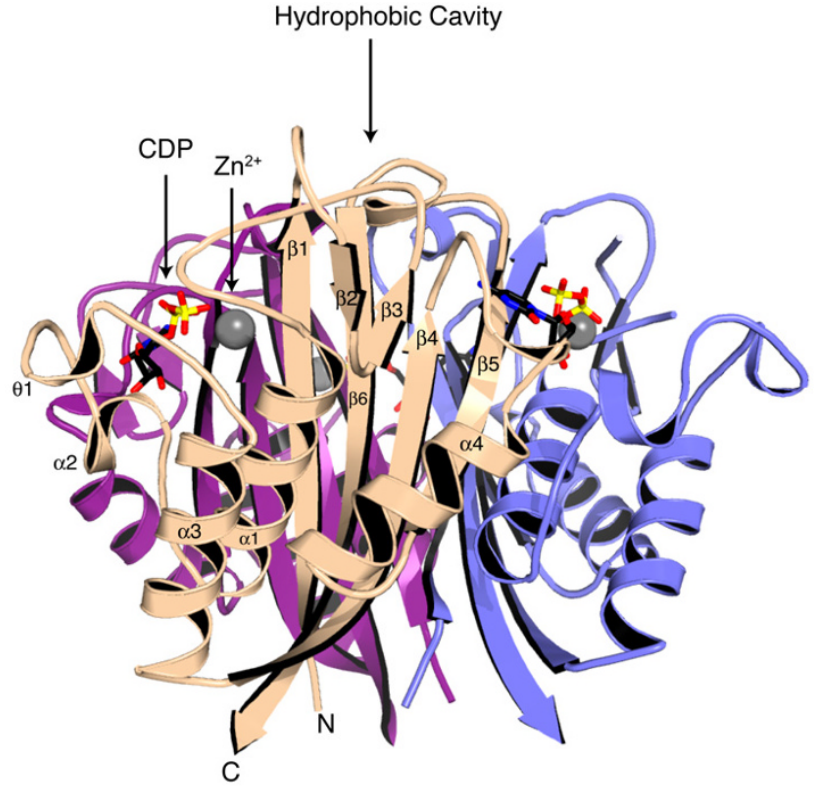

Figure 8

Ribbon diagram of the trimer. Orthogonal view compared to Figure 7.

\section{Active site}

There are three active sites in the trimer, each located at the interface between two adjacent subunits. The active site (Figures 5,6 ) comprises a rigid nucleotide and cation $\left(\mathrm{Zn}^{2+}\right.$ and $\left.\mathrm{Mg}^{2+}\right)$ binding pocket and a flexible loop for binding the ME2P moiety of substrate [16-18,36]. Only one of the two cation-binding sites, the $\mathrm{Zn}^{2+}$ site, is occupied here $[8,15,16]$. This cation is approximately $75 \%$ occupied in two subunits and 50\% occupied in the third. The $\mathrm{Zn}^{2+}$ displays tetrahedral coordination, in similar fashion to that observed in other IspF structures, by Asp11, His13, His45, and the $\beta$-phosphate of CDP. In the higher resolution model of EcIspF, the second cation $\left(\mathrm{Mg}^{2+}\right.$ or $\left.\mathrm{Mn}^{2+}[16,18]\right)$ is coordinated by the side chain of Glu135 and two oxygens from the diphosphate of CDP. In MsIspF and MtIspF, the glutamate is replaced with aspartate. This residue is strictly conserved as an aspartate or glutamate across 450 IspF sequences (data not shown), suggesting that a negative charge is required to coordinate the second cation and that either negatively charged amino acid will suffice. In the structure of MsIspF, the lower resolution data or CDP disorder may preclude identification of the second cation (see below).

Two conformers of CDP, each at approximately half occupancy, are present in each of the three active sites of the trimer. We only show one conformer in Figures 5 and 6 for the purpose of clarity. In the conformers, the ligandprotein interactions are maintained for the pyrimidine and the ribose but diverge at the diphosphate. The average 
thermal parameters of the conformers, hereby referred to as CDP-A and CDP-B, are 20.8 and $29.2 \AA^{2}$, respectively. The presence of CDP disorder is likely linked to the incompletely occupied $\mathrm{Zn}^{2+}$ binding site. The mode of ligand binding of CDP-B more closely resembles that observed in EcIspF (Figure 6). In this mode, three interactions are present between the protein and ligand diphosphate. Two of these are hydrogen bonds formed between the $\alpha$-phosphate and the side chain hydroxyl and main chain amide of the strictly conserved Thr133. The third is ligand-metal ion coordination between the $\beta$-phosphate and the active site $\mathrm{Zn}^{2+}$. The interaction between the $\mathrm{Zn}^{2+}$ and the $\beta$-phosphate is preserved in CDP-A, but an additional hydrogen bond occurs between the $\beta$-phosphate and the hydroxyl group of Thr132. In CDP-A, the $\alpha$-phosphate also forms a hydrogen bond with the side chain hydroxyl of Thr133, but the bridging diphosphate oxygen interacts with the main chain amide of this residue and the side chain of Thr132 instead.

The architecture of the active site at the cytosine and $\mathrm{Zn}^{2+}$ binding sites and the interactions formed with CDP by MsIspF are similar to that observed in EcIspF. Furthermore, MsIspF residues that contribute to this binding site are all identical or conserved in MtIspF (Figure 4). The cytosine is bound in an aliphatic pocket created by side chains of residues from $\beta 5$ and the loop between $\beta 4$ and $\theta 2$ from a single subunit. The cytidine is stacked between the side chains of Ala131 and Lys107, which are strictly conserved in E. coli and M. tuberculosis. Both binding sites in $E c I s p F$ and $M s I s p F$ are characterized by four hydrogen bonds between the pyrimidine and main chain atoms of the protein. In MsIspF, these backbone atoms are from residues Gly103, Pro106, Val108 and Gly109, and, in EcIspF, Ala100, Pro103, Met105, and Leu106. These residues are strictly conserved in MsIspF and MtIspF with the exception that MtIspF Ile109 replaces MsIspF Val108 (Figure 4).

Because the interactions involve backbone atoms, high conservation of these residues is not necessarily required. The critical elements required to maintain similar proteinligand interactions are the shape and size of the cytosine pocket. Two pairs of hydrophobic interactions contribute to this function in MsIspF. One pair of hydrophobic interactions occurs between the side chains of Pro106 and Leu146 and the second between the side chains of Val101 and Val108. Both sets of residues are highly conserved (>85\%) in 450 IspF sequences, including both E. coli and M. tuberculosis. The first is conserved as a proline-leucine/ isoleucine pair and the second as two aliphatic residues, where the identities of the residues are leucine, methionine, valine, isoleucine, or phenylalanine.
The ribose hydroxyls are oriented by several hydrophilic interactions involving strictly conserved residues in $M s I s p F, E c I s p F$ and MtIspF. The ribose hydroxyls form hydrogen bonds with the side chain of Asp59* (the asterisk denotes contributions from another subunit) and the amide of Gly61*, and solvent-mediated interactions are observed with the side chain of Asp49* and the carbonyl of Ala131 (residues Asp56*, Gly58*, Asp46* and Ala131 in $E c I s p F$, respectively). Moreover, in MsIspF and EcIspF, the side chain orientation of Asp59* is maintained through hydrophilic interactions. Here, this aspartate accepts hydrogen bonds donated by amides of Gly $61 *$, Thr62*, and Ala131 and the side chain of Thr62*, and, in EcIspF, with the amides of Gly58*, Lys59*, and Ala131. The MsIspF residues that contribute to the orientation of Asp59* are strictly conserved in the sequence of MtIspF except for Thr62*, which is a glutamate in the latter. Main chain atoms are the primary contributors to stabilization of Asp59*, so this amino acid replacement is unlikely to affect conformation or function.

The nucleotide-binding pocket is only part of the active site. In EcIspF the remaining fragment of substrate, ME2P, is bound by contributions from $\alpha 2, \alpha 3$, and residues 3337 , and a flexible loop, which comprises residues 61-71 [16-18]. The largest $\mathrm{C} \alpha$ r.m.s.d. differences between EcIspF and MsIspF occur in this loop. In EcIspF, the loop is stabilized by hydrogen bonds between the side chain of His $34^{*}$ and the carbonyl atoms of Asp63* and Asp65*. His $34^{*}$ is conserved in MsIspF (His $37^{*}$ ), but the aspartates are not. Here, no well-defined density is observed for His $37^{*}$ in two of the trimer subunits. In the third, the side chain of this residue forms hydrogen bonds to the main chain carbonyl of $\operatorname{Arg} 68^{*}$ and the side chain of Asp67*. The former resembles the EcIspF His $34^{*}$-Asp 65* interaction, but the latter reflects the different conformations of this loop present in the two orthologues. This loop is further stabilized in MsIspF by a hydrophilic interaction between the carbonyl of Ile63* and the side chain of Arg68*, a residue which is not conserved in EcIspF. The stabilization of the loop through hydrogen bonding to the side chain of an aspartate as observed in MsIspF can be maintained in MtIspF as this residue is identical, but the arginine is replaced by a second aspartate. Although the main chain interactions might be preserved by an aspartate, the side chain interactions could not.

ME2P is oriented by several hydrophobic and hydrophilic interactions with EcIspF [18]. The amides of Ser35* and His $34^{*}$ and the hydroxyl of Ser $35^{*}$ form hydrogen bonds with oxygens of the attacking 2-phosphate group. The identities of these residues and the positions of the residues that bind and orient the attacking 2-phosphate group are maintained in MsIspF (Figure 4). In EcIspF, the side chains of Ile57* and Leu76* make van der Waals con- 
tacts with the methyl group of ME2P. These residues are replaced by another hydrophobic pair, Leu60* and Met78*, in MsIspF. When a model of EcIspF containing substrate [PDB code 1U43, [18]] is superimposed onto MsIspF, these residues are able to maintain contact with the methyl group of ME2P. The 3-hydroxyl group of the ligand interacts with the carbonyl of Phe61 in EcIspF. This residue is part of the flexible loop, and the equivalent residue in MsIspF (Phe64*) does not maintain this interaction in the superposition. Phe6 $4^{*}$ is preceded by a glycine in MsIspF and a proline in EcIspF. Glycine flexibility would permit a conformational change to accommodate interactions between Phe64* and the ligand. Alternatively, there is a hydrogen bond present between the carbonyl of Gly65* and the 3-hydroxyl group of the superimposed substrate. The aforementioned residues corresponding to those observed in MsIspF are all identical in MtIspF except for Leu60*, which is an isoleucine instead. The binding component of this residue, the side chain hydrophobicity, is maintained in MtIspF, as this residue is also an isoleucine in E. coli and is strictly conserved as isoleucine, leucine, or valine in $450 \mathrm{IspF}$ sequences.

\section{Conclusion}

There is an urgent need to identify new targets and to develop new treatments for tuberculosis. Our work demonstrates that ispF is essential in M. tuberculosis, thus establishing it as a potentially valuable target for chemotherapeutic intervention. In addition, we have determined the crystal structure of the closely related orthologue MsIspF bound to CDP. The protein is a homotrimer with three equivalent active sites formed at the subunit interfaces. Each active site bears a strong resemblance to those observed in other IspF structures, presenting a rigid $\mathrm{CDP}-\mathrm{Zn}^{2+}$ binding-pocket and a flexible substratebinding loop. MtIspF and MsIspF share $73 \%$ sequence identity, and, of the eleven residues in the active site that bind CDP, ten are identical and the eleventh highly conserved. Based on the high degree of similarity between the orthologues, particularly in the active site, the structure of MsIspF provides a suitable template for structure-based inhibitor design targeting the pathogenic organism $M$. tuberculosis.

\section{Methods \\ Culture and manipulation of $M$. tuberculosis and $M$. smegmatis}

M. tuberculosis (H37Rv) was grown on Middlebrook 7H10 agar or Middlebrook 7H9 broth (with $0.05 \%$ Tween 80 ), with $10 \%$ OADC (oleic acid, bovine serum albumin, dextrose, catalase) supplement (Becton Dickinson). M. smegmatis (ATCC 700084) was grown on Lemco medium (5 g $\mathrm{l}^{-1}$ Lemco powder, $5 \mathrm{~g} \mathrm{l}^{-1} \mathrm{NaCl}, 10 \mathrm{~g} \mathrm{l}^{-1}$ Bacto peptone) with $0.05 \%$ Tween 80 (liquid) or $15 \mathrm{~g} \mathrm{l}^{-1}$ agar (solid).

\section{Plasmids for M. tuberculosis ispF knockouts}

The deletion delivery vector was constructed as follows: PCR was used to amplify the regions either side of ispF using the primer pairs IspFNFor/IspFNRev and IspFCFor/ IspFCRev (Table 1) and the resulting products were subcloned with the Zero Blunt ${ }^{\circledR} \mathrm{TOPO}^{\circledR} \mathrm{PCR}^{\mathrm{C}}$ Cloning Kit (Invitrogen). The DNA fragments were gel purified (Qiagen Qiaquick Gel Extraction Kit) and then cloned into p2NIL [29] to generate a deletion of ispF in which 379 bp of the gene was absent. The marker gene cassette from pGOAL19 [29] was then cloned into the unique PacI site to generate the final delivery vector, p2NIL- $\Delta$ ispF.

To make the complement vector (pAPA3-ispDF) used to generate the merodiploid strain, part of the operon, which includes both the ispD and ispF genes, was amplified by PCR using the primer pair IspDFSh/IspDFRev (Table 1) and subcloned as PacI fragments into the integrating vector pAPA3 [28]. The integrity and directionality of all constructs were confirmed by DNA sequencing.

\section{Isolation and genotyping of recombinant strains}

A single crossover strain was generated by electroporating M. tuberculosis with $1 \mu \mathrm{g}$ plasmid DNA and recombinants selected on $100 \mu \mathrm{g} / \mathrm{ml}$ hygromycin, $20 \mu \mathrm{g} / \mathrm{ml}$ kanamycin and $50 \mu \mathrm{g} / \mathrm{ml} \mathrm{X-gal} \mathrm{as} \mathrm{previously} \mathrm{described} \mathrm{[26].} \mathrm{A} \mathrm{single}$ strain was streaked out in the absence of any antibiotics to allow the second crossover to occur. Double crossovers were selected and screened for using $2 \% \mathrm{w} / \mathrm{v}$ sucrose and $50 \mu \mathrm{g} / \mathrm{ml} \mathrm{X-gal;} \mathrm{white} \mathrm{colonies} \mathrm{were} \mathrm{patch} \mathrm{tested} \mathrm{for} \mathrm{kan-}$ amycin and hygromycin sensitivity to ensure that they had lost the plasmid during homologous recombination. PCR was used to determine the presence of the wild type or deletion allele using primers IspFintA and IspFintB (Table 1), which amplify $1.4 \mathrm{kbp}$ and $1 \mathrm{kbp}$ fragments from the wild type and deletion alleles respectively.

Table I: Primers used in this study

\begin{tabular}{ll}
\hline Primer & 5' Sequence* \\
\hline IspFNFor & AAGCTTgtacgagttcccgctgaaacgc \\
IspFNRev & GGATCCgagagtctgcccgtcgagctg \\
IspFCFor & GGATCCgcaatcgctacggcattggtggt \\
IspFCRev & GGTACCaccaccaccgacgggctgggc) \\
IspDFSh & TTAATTAAgacgccaaagccgagaccatcctt \\
IspDFRev & TTAATTAAgccagcttacctgcccaattgctg \\
IspFIntA & ggtcgaatcgcactgacac \\
IspFIntB & cgatcatctgggtgatatgc \\
MsIspFNter & CATATGatgttgcctcgcgtagggc \\
MslspFCter & GGATCCtactttgcatcaccgaccggtt \\
IspDUSFor & gacgagaatcaatgagacct \\
IspDUSRev & agtgatatcggctcggtgac \\
\hline
\end{tabular}

*Upper case letters indicate inserted restriction sites. 
To generate the merodiploid strain, the pAPA3-ispDF plasmid was electroporated into the single crossover strain and recombinants isolated on $10 \mu \mathrm{g} / \mathrm{ml}$ gentamicin, 100 $\mu \mathrm{g} / \mathrm{ml}$ hygromycin, $20 \mu \mathrm{g} / \mathrm{ml}$ kanamycin and $50 \mu \mathrm{g} / \mathrm{ml} \mathrm{X-}$ gal. A single recombinant was streaked out without antibiotics to allow a second crossover to occur, and double crossovers were isolated as before, except that gentamicin was included at all stages. PCR and Southern blot analysis were used to confirm the double crossover deletion allele (delinquent mutant) generated from the merodiploid strain.

\section{Southern analysis}

To generate a probe for Southern analysis, the region upstream of the is $p D$ was PCR-amplified using primers IspDUSFor and IspDUSRev (Table 1) and the isolated fragment labeled with AlkPhos Direct system (GE Healthcare). Genomic M. tuberculosis DNA ( $2 \mu \mathrm{g})$ was digested with BamHI; the digestion products were separated on an agarose gel and transferred by vacuum blotter onto a Hybond $\mathrm{N}+$ membrane (GE Healthcare). The membrane was hybridized for $16 \mathrm{~h}$ in Alk Phos Direct hybridization buffer (GE Healthcare) at $65^{\circ} \mathrm{C}$ with the labeled probe. Primary and secondary post hybridization washes were carried out (two primary washes for $10 \mathrm{~min}$ each at $55^{\circ} \mathrm{C}$ and two secondary for 5 min each at RT, as per manufacturers' instructions), and the probe detected by CDP-Star (GE Healthcare).

\section{Cloning and expression of $M$. smegmatis ispF}

The ispF gene was amplified by PCR from genomic DNA, previously obtained with an established protocol [38], using the primers MsIspFNter and MsIspFCter (Table 1) and cloned into NdeI/BamHI-digested pET15b_TEV, a modified pET15b (Novagen) expression vector that includes an $\mathrm{N}$-terminal tobacco etch virus (TEV) protease cleavage site in place of the thrombin cleavage site. The integrity of the pET15b_TEV-ispF construct was confirmed by sequencing.

This construct was chemically transformed into BL21(DE3) Gold cells (Stratagene) and selected for on Luria-Bertani (LB) agar plates containing carbenicillin (50 $\mu \mathrm{g} / \mathrm{ml}$ ). A single colony was cultured at $37^{\circ} \mathrm{C}$ to an $A_{600}$ of $\sim 0.6$ in $1 \mathrm{~L}$ of LB containing carbenicillin $(50 \mu \mathrm{g} / \mathrm{ml})$ and transferred to an ice water bath for 20 minutes. Subsequently, $1 \mathrm{mM}$ isopropyl $\beta$-D-1-thiogalactopyranoside was added to induce expression and the culture was incubated at $22^{\circ} \mathrm{C}$ overnight. Cells were harvested by centrifugation and stored at $-20^{\circ} \mathrm{C}$.

\section{Purification of MslspF}

The cell pellet was resuspended in $30 \mathrm{~mL}$ of binding buffer (500 mM NaCl, $20 \mathrm{mM}$ Tris-HCl, pH 8, 15 mM imidazole) containing lysozyme and DNAse I [16] and lysed using a One-shot cell disruptor (Constant Cell Disruption Systems). The soluble fraction was isolated by centrifugation $\left(48,400 \mathrm{~g}, 30\right.$ minutes at $\left.4{ }^{\circ} \mathrm{C}\right)$, passed through a 0.2 $\mu$ filter, and loaded onto a $5 \mathrm{~mL}$ HisTrap HP column (GE Healthcare) loaded with $\mathrm{Ni}^{2+}$ and equilibrated in binding buffer. The protein was eluted using a combination of step and linear gradients from 0 to $500 \mathrm{mM}$ imidazole and concentrated to $873 \mu \mathrm{M}$ (theoretical $\varepsilon_{0}=6990 \mathrm{M}^{-1} \mathrm{~cm}^{-1}$ including the His-tag). The His-tag was cleaved with TEV protease ( $2 \mathrm{mg}$ at $22^{\circ} \mathrm{C}$ for 12 hours). The sample was subsequently dialyzed into $50 \mathrm{mM} \mathrm{NaCl}, 20 \mathrm{mM}$ Tris$\mathrm{HCl}, \mathrm{pH} 8$ and $1 \mathrm{mM}$ dithiothreitol, passed over a $5 \mathrm{~mL}$ HisTrap HP column to remove TEV protease and uncleaved protein, and further purified by anion exchange chromatography (5 mL Q HP, GE Healthcare). The sample was then dialyzed into $50 \mathrm{mM} \mathrm{NaCl}, 10 \mathrm{mM}$ Tris- $\mathrm{HCl}$, $\mathrm{pH} 8,2 \mathrm{mM} \mathrm{MgCl}_{2}$ and concentrated to $405 \mu \mathrm{M}$ (theoretical $\varepsilon_{0}$ of $5500 \mathrm{M}^{-1} \mathrm{~cm}^{-1}$ excluding the His-tag). This protein solution was used for crystallization. The high degree of sample purity was confirmed by SDS-PAGE and matrixassisted laser desorption ionization-time-of-flight mass spectrometry.

\section{Crystallization and data collection}

Prior to crystallization, the protein was incubated with 5 $\mathrm{mM}$ CDP at $4{ }^{\circ} \mathrm{C}$ for 12 hours. Crystals were grown in three days by sitting drop vapor diffusion at $20^{\circ} \mathrm{C}$ using $0.8 \mu \mathrm{L}$ of protein solution and $0.8 \mu \mathrm{L}$ of reservoir $(18 \%$ PEG 8000, $0.1 \mathrm{M}$ sodium cacodylate $\mathrm{pH} 6.5,0.2 \mathrm{M}$ calcium acetate). A single crystal $(50 \times 50 \times 50 \mu \mathrm{m})$ was cryoprotected in reservoir adjusted to include $18 \%$ glycerol and flash-cooled at $-173^{\circ} \mathrm{C}$. Diffraction data were collected (Table 2) at the European Synchrotron Radiation Facility (ESRF), station ID 23-2, on a MarMosaic 225 CCD detector at a wavelength of $0.8730 \AA$. The data were integrated, merged, and scaled using MOSFLM [39] and SCALA [40] from the CCP4 suite of programs [41].

\section{Structure determination and refinement}

The crystal belongs to space group I4 and has three subunits in the asymmetric unit. The structure was solved by molecular replacement with AMORE [42] using an EcIspF trimer as the search model [PDB code 1GX1, [16]]. EcIspF shares 38\% amino acid identity with MsIspF. Search model bias was removed/reduced with prime-and-switch phasing and a partial MsIspF model was built using RESOLVE [43]. Restrained maximum likelihood refinement was done using REFMAC5 [44] and PRODRG [45] to provide ligand dictionaries. Non-crystallographic symmetry restraints were imposed early on but removed at later stages of refinement. The $\mathrm{R}_{\text {free }}$ calculation was performed on $5 \%$ of the data. COOT [46] was used to inspect Fourier syntheses and manipulate the model during refinement. The occupancies for CDP and $\mathrm{Zn}^{2+}$ were based on consideration of refined thermal parameters and the 
Table 2: Crystallographic statistics

\begin{tabular}{ll}
\hline Structure & M. smegmatis IspF \\
\hline Unit cell, a, $b, c(\AA)$ & $159.1,159.1,54.2$ \\
Resolution range $(\AA)$ & $51.3-2.2 \AA$ \\
No. of observations & 117,125 \\
No. of unique reflections & 33,193 \\
Completeness $(\%)$ & $95.4(76.3)^{\dagger}$ \\
$</ / \sigma(I)>$ & $6.0(1.7)$ \\
$\mathrm{R}_{\text {sym }}(\%)$ & $8.1(34.6)$ \\
Multiplicity & $3.5(2.1)$ \\
Wilson $B\left(\AA^{2}\right) / D P I(\AA) \ddagger$ & $28.1 / 0.16$ \\
Protein residues & 461 \\
Water molecules & 272 \\
CDP/Zn ${ }^{2+}$ molecules (occupancy) & $3(1) / 3(0.75 / 0.5 / 0.75)$ \\
IPP molecules (occupancy) & $1(0.5)$ \\
PEG/Glycerol/Ethylene glycol/Acetate & $2 / 3 / 2 / 1$ \\
molecules & \\
$\mathrm{R}_{\text {work }} / \mathrm{R}_{\text {free }}(\%)$ & $16.3 / 20.6$ \\
Average $B\left(\AA^{2}\right)$ & \\
Subunit A/B/C overall & $30.7 / 19.7 / 3 I .8$ \\
All main/side chain & $25.9 / 29.3$ \\
Waters & 35.8 \\
CDP/Zn ${ }^{2+}$ & $25.0 / 36.0$ \\
IPP & 47.5 \\
PEG/Glycerol/Ethylene glycol/Acetate & $45.3 / 58.4 / 28.5 / 38.9$ \\
RMS bond lengths $(\AA)$ & 0.013 \\
RMS bond angles $\left({ }^{\circ}\right)$ & 1.570 \\
PDB Code & $2 U Z \mathrm{H}$ \\
&
\end{tabular}

† Values in parentheses pertain to the highest resolution shell (width $=0.11 \AA$ ). $B$ is the isotropic thermal parameter.

$\ddagger \mathrm{DPI}=$ diffraction-component precision index [53].

appearance of electron and difference density maps. Statistics for the model are presented in Table 2.

\section{Model analysis}

Root-mean-square deviation (r.m.s.d.) values for superpositions were calculated using LSQMAN [47]. The values for the superpositions of chain $\mathrm{A}$ onto $\mathrm{B}$, A onto $\mathrm{C}$, and $\mathrm{B}$ onto C, respectively, were $0.54 \AA, 0.59 \AA$, and $0.67 \AA$ over $153 \mathrm{C} \alpha$ atoms. Analysis of model geometry with PROCHECK [48] demonstrated that all residues are within allowed regions of the Ramachandran plot. Secondary structure assignments were made using DSSP [49], COOT, and by visual inspection. The trimer interface was analyzed using the Protein-Protein Interaction Server [50]. The volumes of the hydrophobic cavities at the trimer centers were calculated and refined with VOIDOO [51] using a rolling probe with a radius of $1.4 \AA$. Figure 1 was created with ChemDraw, Figure 2 with Adobe Illustrator and Photoshop, 4 with Aline (C. S. Bond and A. W. Schüttelkopf, personal communication), and 5-8 with PyMOL [52].

\section{Authors' contributions}

LB carried out cloning, biochemical and crystallographic experiments, ACB carried out all research on M. tuberculo- sis. TP planned and supervised all work with $M$. tuberculosis. WNH conceived of the study, and together all authors participated in its design and coordination. All authors contributed to data interpretation and in writing the manuscript.

\section{Acknowledgements}

We thank D. Flot at the ESRF, C. Bond, A. Schüttelkopf and members of the labs for help and advice, Dina Robertson and Scott Cameron for technical assistance. This research was supported by EU Project QLRT-200I00887, the Biotechnology and Biological Sciences Research Council Project BB/D000I8I, the (Structural Proteomics of Rational Targets) and The Wellcome Trust.

\section{References}

I. World Health Organisation: Global tuberculosis control: surveillance, planning, financing. 2005 [http://www.who.int/mediacen tre/factsheets/fs $104 / \mathrm{en} /$ index.html]. WHO report

2. Swanson KM, Hohl RJ: Anti-cancer therapy: targeting the mevalonate pathway. Curr Cancer Drug Targets 2006, 6:15-37.

3. Jomaa H, Wiesner J, Sanderbrand S, Altincicek B, Weidemeyer C, Hintz M, Turbachova I, Eberl M, Zeidler J, Lichtenthaler HK, Soldati $D$, Beck E: Inhibitors of the nonmevalonate pathway of isoprenoid biosynthesis as antimalarial drugs. Science 1999, 285: I573-1576.

4. Dewick PM: Medicinal natural products: a biosynthetic approach. 2nd edition. Wiley, Chichester, West Sussex, England; New York, NY, USA; 2002

5. Crick DC, Schulbach MC, Zink EE, Macchia M, Barontini S, Besra GS, Brennan PJ: Polyprenyl phosphate biosynthesis in Mycobacterium tuberculosis and Mycobacterium smegmatis. J Bacteriol 2000, 182:577I-5778.

6. Koga $\mathrm{Y}$, Morii H: Biosynthesis of ether-type polar lipids in archaea and evolutionary considerations. Microbiol Mol Biol Rev 2007, 71:97-I 20.

7. Eisenreich W, Schwarz M, Cartayrade A, Arigoni D, Zenk MH, Bacher $A$ : The deoxyxylulose phosphate pathway of terpenoid biosynthesis in plants and microorganisms. Chem Biol 1998, 5:R22I-233.

8. Hunter WN: Structure and reactivity in the non-mevalonate pathway of isoprenoid precursor biosynthesis. I Biol Chem 2007, 282:21573-1577.

9. Kuzuyama $\mathrm{T}$ : Mevalonate and nonmevalonate pathways for the biosynthesis of isoprene units. Biosci Biotechnol Biochem 2002, 66:1619-1627.

10. Lichtenthaler HK, Schwender J, Disch A, Rohmer M: Biosynthesis of isoprenoids in higher plant chloroplasts proceeds via a mevalonate-independent pathway. FEBS Lett 1997, 400:27I-274.

II. Schwender J, Seemann M, Lichtenthaler HK, Rohmer M: Biosynthesis of isoprenoids (carotenoids, sterols, prenyl side-chains of chlorophylls and plastoquinone) via a novel pyruvate/glyceraldehyde 3-phosphate non-mevalonate pathway in the green alga Scenedesmus obliquus. Biochem J 1996, 316:73-80.

12. Borrmann S, Lundgren I, Oyakhirome S, Impouma B, Matsiegui PB, Adegnika AA, Issifou S, Kun JF, Hutchinson D, Wiesner J, Jomaa $H$, Kremsner PG: Fosmidomycin plus clindamycin for treatment of pediatric patients aged I to 14 years with Plasmodium falciparum malaria. Antimicrob Agents Chemother 2006, 50:27I3-27I8.

13. Henriksson LM, Unge T, Carlsson J, Aqvist J, Mowbray SL, Jones TA: Structures of Mycobacterium tuberculosis I-deoxy-D-xylulose-5-phosphate reductoisomerase provide new insights into catalysis. J Biol Chem 2007, 282:19905-19916.

14. Rohdich F, Wungsintaweekul J, Fellermeier M, Sagner S, Herz S, Kis $\mathrm{K}$, Eisenreich W, Bacher A, Zenk MH: Cytidine 5'-triphosphatedependent biosynthesis of isoprenoids: YgbP protein of Escherichia coli catalyzes the formation of 4-diphosphocytidyl-2-C-methylerythritol. Proc Natl Acad Sci USA 1999, 96: II758-II763.

15. Kemp LE, Alphey MS, Bond CS, Ferguson MA, Hecht S, Bacher A, Eisenreich W, Rohdich F, Hunter WN: The identification of isoprenoids that bind in the intersubunit cavity of Escherichia 
coli 2C-methyl-D-erythritol-2,4-cyclodiphosphate synthase by complementary biophysical methods. Acta Crystallogr D Biol Crystallogr 2005, 6 I (Pt I):45-52.

16. Kemp LE, Bond CS, Hunter WN: Structure of 2C-methyl-Derythritol 2,4- cyclodiphosphate synthase: an essential enzyme for isoprenoid biosynthesis and target for antimicrobial drug development. Proc Natl Acad Sci USA 2002 99:6591-6596.

17. Richard SB, Ferrer JL, Bowman ME, Lillo AM, Tetzlaff CN, Cane DE, Noel JP: Structure and mechanism of 2-C-methyl-D-erythritol 2,4-cyclodiphosphate synthase an enzyme in the mevalonate-independent isoprenoid biosynthetic pathway. J Biol Chem 2002, 277:8667-8672.

18. Steinbacher S, Kaiser J, Wungsintaweekul J, Hecht S, Eisenreich W, Gerhardt S, Bacher A, Rohdich F: Structure of 2C-methyl-Derythritol-2,4-cyclodiphosphate synthase involved in mevalonate-independent biosynthesis of isoprenoids. J Mol Biol 2002, 316:79-88.

19. Campbell TL, Brown ED: Characterization of the depletion of 2C-methyl-D-erythritol-2,4-cyclodiphosphate synthase in Escherichia coli and Bacillus subtilis. I Bacteriol 2002, 184:5609-56/8.

20. Freiberg C, Wieland B, Spaltmann F, Ehlert K, Brotz H, Labischinski $\mathrm{H}$ : Identification of novel essential Escherichia coli genes conserved among pathogenic bacteria. I Mol Microbiol Biotechnol 2001, 3:483-489.

21. Akerley BJ, Rubin EJ, Novick VL, Amaya K, Judson N, Mekalanos JJ: A genome-scale analysis for identification of genes required for growth or survival of Haemophilus influenzae. Proc Natl Acad Sc USA 2002, 99:966-971.

22. Sassetti CM, Boyd DH, Rubin E): Genes required for mycobacterial growth defined by high-density mutagenesis. Mol Microbio 2003, 48:77-84.

23. Crane CM, Kaiser J, Ramsden NL, Lauw S, Rohdich F, Eisenreich W Hunter WN, Bacher A, Diederich F: Fluorescent inhibitors for IspF, an enzyme in the non-mevalonate pathway for isoprenoid biosynthesis and a potential target for antimalarial therapy. Angew Chem Int Ed Engl 2006, 45: I069-1074.

24. Illarionova $\mathrm{V}$, Kaiser J, Ostrozhenkova E, Bacher A, Fischer M, Eisenreich W, Rohdich F: Nonmevalonate terpene biosynthesis enzymes as antiinfective drug targets: substrate synthesis and high-throughput screening methods. J Org Chem 2006, 71:8824-8834.

25. Parish T, Gordhan BG, McAdam RA, Duncan K, Mizrahi V, Stoker NG: Production of mutants in amino acid biosynthesis genes of Mycobacterium tuberculosis by homologous recombination. Microbiology 1999, I45(Pt I 2):3497-3503.

26. Parish T, Lewis J, Stoker NG: Use of the mycobacteriophage L5 excisionase in Mycobacterium tuberculosis to demonstrate gene essentiality. Tuberculosis 200I, 8 I:359-364.

27. Parish T, Roberts G, Laval F, Schaeffer M, Daffe M, Duncan K: Functional complementation of the essential gene fabG I of Mycobacterium tuberculosis by Mycobacterium smegmatis fabG, but not Escherichia coli fabG. J Bacteriol 2007, 189:372I-3728.

28. Parish T, Stoker NG: glnE is an essential gene in Mycobacterium tuberculosis. I Bacteriol 2000, 182:57/5-5720.

29. Parish T, Stoker NG: Use of a flexible cassette method to generate a double unmarked Mycobacterium tuberculosis tlyA plcABC mutant by gene replacement. Microbiology 2000, I 46: 1969-1975.

30. Abagyan R, Batalov S, Cardozo T, Totrov M, Webber J, Zhou Y: Homology modeling with internal coordinate mechanics: deformation zone mapping and improvements of models via conformational search. Proteins 1997:29-37.

31. Martin AC, MacArthur MW, Thornton JM: Assessment of comparative modeling in CASP2. Proteins 1997:14-28.

32. Thiry A, Ledecq M, Cecchi A, Dogne JM, Wouters J, Supuran CT, Masereel $B$ : Indanesulfonamides as carbonic anhydrase inhibitors Toward structure-based design of selective inhibitors of the tumor-associated isozyme CA IX. I Med Chem 2006, 49:2743-2749.

33. Takami A, Iwakubo M, Okada $\mathrm{Y}$, Kawata T, Odai H, Takahashi N, Shindo K, Kimura K, Tagami Y, Miyake M, Fukushima K, Inagaki M, Amano $M$, Kaibuchi $K$, lijima $H$ : Design and synthesis of Rho kinase inhibitors (I). Bioorg Med Chem 2004, 1 2:21 I5-37.
34. Gabrielsen M, Bond CS, Hallyburton I, Hecht S, Bacher A, Eisenreich W, Rohdich F, Hunter WN: Hexameric assembly of the bifunctional methylerythritol 2,4-cyclodiphosphate synthase and protein-protein associations in the deoxy-xylulose-dependent pathway of isoprenoid precursor biosynthesis. J Biol Chem 2004, 279:52753-5276I.

35. Kishida H, Wada T, Unzai S, Kuzuyama T, Takagi M, Terada T, Shirouzu M, Yokoyama S, Tame JR, Park SY: Structure and catalytic mechanism of 2-C-methyl-D-erythritol 2,4-cyclodiphosphate (MECDP) synthase, an enzyme in the non-mevalonate pathway of isoprenoid synthesis. Acta Crystallogr D Biol Crystallogr 2003, 59(Pt I):23-3I.

36. Lehmann C, Lim K, Toedt J, Krajewski W, Howard A, Eisenstein E, Herzberg O: Structure of 2C-methyl-D-erythrol-2,4-cyclodiphosphate synthase from Haemophilus influenzae: activation by conformational transition. Proteins 2002, 49:135-138.

37. Ni S, Robinson H, Marsing GC, Bussiere DE, Kennedy MA: Structure of 2C-methyl-D-erythritol-2,4-cyclodiphosphate synthase from Shewanella oneidensis at I.6 ̊̊: identification of farnesyl pyrophosphate trapped in a hydrophobic cavity. Acta Crystallogr Biol Crystallogr 2004, D60:1949-1957.

38. Wilson K: Preparation of genomic DNA from bacteria. In CurrentProtocols in Molecular Biology Edited by: Ausubel FM. Greene Publishing Associates, Brooklyn, New York; 1994:2.4.I-2.4.2.

39. Leslie AGW: Recent changes to the MOSFLM package for processing film and image plate data. Joint CCP4 and ESF-EAMCB Newsletter on Protein Crystallography 1992:26-30.

40. Evans PR: Scaling of MAD data. Joint CCP4 ESF-EAMCB Newsletter on Protein Crystallography 1997, 33:22-24.

41. CCP4: The CCP4 suite: programs for protein crystallography. Acta Crystallogr Biol Crystallogr 1994, 50:760-763.

42. Navaza J: AMoRe: an automated package for molecular replacement. Acta Crystallogr 1994, A50:157-163.

43. Terwilliger TC: Maximum-likelihood density modification. Acta Crystallogr Biol Crystallogr 2000, D56:965-972.

44. Murshudov GN, Vagin AA, Dodson EJ: Refinement of macromolecular structures by the maximum-likelihood method. Acto Crystallogr Biol Crystallogr 1997, D53:240-255.

45. Schüttelkopf AW, van Aalten DM: PRODRG: a tool for highthroughput crystallography of protein-ligand complexes. Acta Crystallogr Biol Crystallogr 2004, D60: I 355-I 363.

46. Emsley P, Cowtan K: Coot: model-building tools for molecular graphics. Acta Crystallogr Biol Crystallogr 2004, D60:2 I 26-2 I 32

47. KleywegT G]: Use of non-crystallographic symmetry in protein structure refinement. Acta Crystallogr Biol Crystallogr 1996, D52:842-57.

48. Laskowski RA, MacArthur MW, Moss DS, Thornton JM: PROCHECK: a program to check the stereochemical quality of protein structures. J App Cryst 1993, 283:283-29I.

49. Kabsch W, Sander C: Dictionary of protein secondary structure: Pattern recognition of hydrogen-bonded and geometrical features. Biopolymers 1983, 22:2577-2637.

50. Jones S, Thornton JM: Principles of protein-protein interactions. Proc Natl Acad Sci USA 1999, 93: I 3-20.

51. Kleywegt GJ, Jones TA: Detection, delineation, measurement and display of cavities in macromolecular structures. Acta Crystallogr Biol Crystallogr 1994, D50: 178- 185

52. DeLano WL: The PyMOL Molecular Graphics System. DeLano Scientific, San Carlos, CA; 2002.

53. Cruickshank DW: Remarks about protein structure precision. Acta Crystallogr Biol Crystallogr 1999, D55:583-601. 